\title{
Optimization of Pilot Symbol-Assisted RAKE Receivers for DS-CDMA Systems
}

\author{
Tao Cui and Chintha Tellambura \\ Department of Electrical and Computer Engineering \\ University of Alberta \\ Edmonton, AB, Canada T6G 2V4 \\ Email: \{taocui, chintha\}@ece.ualberta.ca
}

\begin{abstract}
For optimizing pilot sequences for general wideband direct-sequence (DS) code division multiple access (CDMA) systems in a slow fading Rayleigh channel, we derive a design criterion by minimizing the mean square error (MSE) of the channel estimate. We analyze the effects of imperfect channel estimation (CE) on CDMA system based on maximal ratio combining (MRC) RAKE receiver in both Uniform Power Delay Profile (UPDP) and Non-uniform Power Delay Profile (NPDP) channels. Published results on the effect of $\mathrm{CE}$ errors hold only for UPDP channels. We therefore use a characteristic function method to derive new closed-form expressions for the BER of RAKE receivers in NPDP channels. Constraining the energy per data frame to be constant, we optimize the length of the pilot symbols by minimizing the bit error rate (BER) of the MRC receiver. We show an elegant result that the optimal number of pilot symbols is equal to the square root of the frame length for UPDP channels and for NPDP in high SNR region.
\end{abstract}

\section{INTRODUCTION}

Wideband direct-sequence (DS) code division multiple access (CDMA) with pilot-assisted coherent detection has been proposed for next generation cellular systems [1]. A coherent system requires channel estimation that can track time-varying mobile radio channels. For this purpose, pilot symbols are transmitted along with data symbols. If the frame energy is constant, power allocation to pilot symbols reduces the power available for data symbols, which may increase the BER. However, increased pilot symbol energy improves the accuracy of the channel estimates and thereby improves the BER. These two counteracting tendencies ensure that there is an optimal allocation of energy for the pilot symbols.

Many proposed RAKE receivers in the literature employ coherent detection with MRC, or differential detection with equal gain combining. It is well known that coherent detection of binary phase-shift keying (BPSK) provides a $3 \mathrm{~dB}$ gain over differential detection in flat fading channels. Hence, coherent detection with maximal ratio combing is preferable if accurate $\mathrm{CE}$ is possible. However, the CE accuracy is limited by the pilot symbols to the noise power ratio. The effects of $\mathrm{CE}$ errors on the performance of MRC systems have only been analyzed in UPDP channels. In [2], [3], the effect of Gaussian estimation errors on MRC, which are independent of the additive channel noise, is investigated. Proakis [4, p.954] derives the BER of M-ary phase-shift keying (MPSK). Singh et al. [5] analyze a Rake case in which CE errors are not independent of the additive noise. To the best of our knowledge, the effects of CE errors on the performance of MRC systems in NPDP channels have not been studied. Thus the BER in NPDP channels is of great practical and theoretical importance.

We analyze the RAKE receiver with MRC and imperfect channel estimation in NPDP channels. The well-known results on the impact of CE errors on the BER due to Proakis [4, p.949] hold only for UPDP channels. We derive a novel closed-form expression for the BER with a different approach from [4]. Finally, by assuming the energy is constant within each data block, we optimize the pilot symbols' length by minimizing the BER in both the UPDP and NPDP channels.

The rest of the paper is organized as follows. In Section II, we briefly overview the basic system model. The analysis of the NPDP channel is derived in Section III. We optimize the pilot symbols length in section IV. Computer simulation results are given in Section V and final conclusions are made in Section VI.

Notation: If $x$ and $y$ are Gaussian random variables (RV) with mean $\mu_{x}, \mu_{y}$ and variance $\sigma^{2} / 2$, the $z=x+j y$ has a Complex Gaussian distribution. We write $z \sim \mathcal{C N}\left(\mu_{x}+\right.$ $j \mu_{y}, \sigma^{2}$ ) (where $j=\sqrt{-1}$ ) in this case.

\section{System ModeL}

\section{A. Transmitter}

The transmitter performs spreading and chip pulse shaping. Each data bit is chosen from the alphabet $\{0,1\}$. Each data bit is mapped to -1 or +1 (BPSK). In this paper, we consider serial multiplexing of pilot symbols with the data. A frame consists of $P$ BPSK pilot symbols followed by $N$ BPSK data symbols. The pilot symbols are reserved for channel estimation and are not spread via a spreading sequence. Only data symbols are spread by spreading sequence $c_{n}$, which has ideal autocorrelation properties. The resulting frame passes though a pulse shaping filter $p(t)$, modulates a RF frequency $f_{0}$ and is transmitted through the channel (Fig. 1). The transmitted signal is denoted by $x(n)$. We assume all data symbols have the same energy $E_{b}$ and all the pilot symbols have the same energy $E_{p}$. 


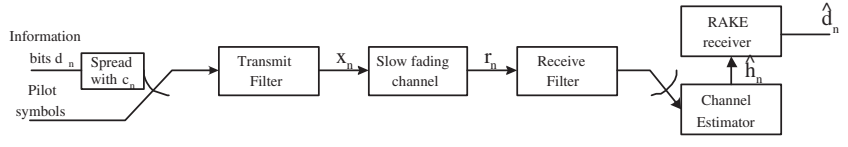

Fig. 1. Block diagram of the pilot symbol assisted communication system

\section{B. Channel Model}

The mobile radio channel is modelled as a frequency selective Rayleigh-fading channel [4]. For convenience, it is assumed that $L$ resolvable propagation paths exist. The lowpass impulse response for a time-invariant frequency selective channel is given by $[4, \mathrm{p} .841]$

$$
h(\tau ; t)=\sum_{k=-\infty}^{\infty} h_{k}(t) \delta(\tau-k / W)
$$

For a total multipath spread of $T_{m}$ and bandwidth $W$, this tapped delay line model can be truncated at $L=\left\lfloor T_{m} W\right\rfloor+1$. At the receiver input, white Gaussian noise $w(n)$ with variance $\sigma_{n}^{2}=N_{0} / 2$ is added, which may model both thermal noise and interference from other users. The channel output is given by

$$
r(n)=\sum_{k=0}^{L-1} x(n-k) h_{k}+w(n)
$$

where the $L$ channel taps $h_{k}=h_{k}(t), k=0,1, \cdots, L-1$ are $h_{k} \sim \mathcal{C N}\left(0, \sigma_{k}^{2}\right)$. For brevity, we consider two power delay profiles: the uniform power delay profile (UPDP) and exponential power delay profile (EPDP). They can be expressed as

$$
\sigma_{k}^{2}=E\left[\left|h_{k}\right|^{2}\right]=\left\{\begin{array}{cc}
C & \text { UPDP } \\
C e^{-\frac{\tau_{0}}{\tau_{r m s}} k} & \text { EPDP }
\end{array}\right.
$$

where $\tau_{0}$ is the time duration between two consecutive discrete taps, $\tau_{r m s}$ is the rms delay spread value and $C$ is a constant term that normalizes the power. However, our analytical results developed in Section IV can handle arbitrary NPDP channels.

\section{Receiver}

The receiver (Fig. 1) comprises a coherent channel estimator and a RAKE demodulator. The receiver is assumed to have perfect frequency synchronization. The channel responses including delay estimation are estimated by the channel estimator via the pilot symbols. We assume that the spreading sequences have ideal correlation properties so that the interference from other users can be ignored and the multipath signals are resolvable.

The decision variables for coherent detection of the binary signals in RAKE receiver can be expressed as [4, p.843]

$$
U_{m}=\operatorname{Re}\left[\sum_{k=0}^{L-1} \hat{h}_{k}^{*} \int_{0}^{T} r(t) c_{n}(t-k / W) d t\right], \quad m=1,2
$$

where $c_{n}(t)=\left(2 c_{n}-1\right) g(t-n / W)$ is the signature waveform of the $n$th user. $g(t)$ represents a pulse of duration $1 / W$ and arbitrary shape.

\section{RAKE RECEIVER BER FOR CDMA WITH IMPERFECT CHANNEL ESTIMATION}

The pilot-aided channel estimate $\hat{\mathbf{h}}$ differs from the true channel by a complex Gaussian error. Therefore $\hat{h}_{l}$ can also be modelled as complex Gaussian with variance $\sigma_{l}^{2}+\eta \sigma_{n}^{2}$, where $\eta$ is the scaling factor determined by pilot symbols. In this section, we derive the BER in both UPDP channels and NPDP channels.

\section{A. UPDP channels}

The BER of the output SNR, $\gamma$, of a MRC with Gaussian distributed weighting errors for BPSK in UPDP has been derived by Proakis [4, p.955] and given by

$$
P(\mu)=\frac{1}{2}\left[1-\mu \sum_{k=0}^{L-1}\left(\begin{array}{c}
2 k \\
k
\end{array}\right)\left(\frac{1-\mu^{2}}{4}\right)^{k}\right]
$$

where $L$ is the length of mobile channel, and $\mu$ is the crosscorrelation coefficient of the receiver correlator output samples and the channel estimates derived from the pilot symbols.

We denote $Y_{k}$ as $\hat{h}_{k}$ and $X_{k}$ as

$$
X_{k}=2 \varepsilon h_{k} s_{n}+N_{k}, \quad n=1,2
$$

where $s_{n}=\exp [j \pi(n-1)]$.

The output of the RAKE receiver can be expressed as

$$
z=\sum_{k=0}^{L-1} X_{k} \hat{h}_{k}^{*}=\sum_{k=0}^{L-1} X_{k} Y_{k}^{*}
$$

Then $\mu$ is given by [4, p.952]

$$
\begin{aligned}
m_{x x}=E\left(\left|X_{k}\right|^{2}\right), m_{y y} & =E\left(\left|Y_{k}\right|^{2}\right), m_{x y}=E\left(X_{k} Y_{k}^{*}\right) \\
\mu & =\frac{m_{x y}}{m_{x x} m_{y y}}
\end{aligned}
$$

Eq. (5) is derived assuming all pairs $\left(X_{k}, Y_{k}\right)$ are independent and identically distributed. The method was due to Proakis [4, p.952]. He derives the joint characteristic function (chf) of the real and imagine parts of $X_{k} Y_{k}^{*}$ and raises the chf to power $L$, Fourier transforms the results, converts rectangular to polar form and finally integrates over the amplitude variable. The result is the pdf of the decision phase variable. However, this approach does not work when $\left(X_{k}, Y_{k}\right)$ are distributed non-identically.

\section{B. NPDP channels}

In this subsection, we derive the BER of RAKE receiver in NPDP channels with a different approach from the UPDP case given by Proakis. We still use the above defined symbols. 
Assuming $s_{1}$ is transmitted, the error probability of BPSK RAKE receiver is the probability that $P(D<0)$, where

$$
\begin{aligned}
D & =\operatorname{Re}\left\{\sum_{k=0}^{L-1} X_{k} Y_{k}^{*}\right\} \\
& =\sum_{k=0}^{L-1} X_{k} Y_{k}^{*}+X_{k}^{*} Y_{k} .
\end{aligned}
$$

This is a special case of the general quadratic form

$$
D=\sum_{k=0}^{L-1}\left(A\left|X_{k}\right|^{2}+B\left|X_{k}\right|^{2}+C X_{k} Y_{k}^{*}+C^{*} X_{k}^{*} Y_{k}\right)
$$

when $A=0, B=0$ and $C=1, X_{k}$ and $Y_{k}$ are a pair of correlated complex Gaussian random variables. The $L$ pairs $\left\{X_{k}, Y_{k}\right\}$ are mutually statistically independent.

From [4, p.943], the probability of error is given by

$$
\begin{aligned}
P_{b} & =P(D<0)=\int_{-\infty}^{0} p(D) d D \\
& =-\frac{1}{2 \pi j} \int_{-\infty+j \varepsilon}^{+\infty+j \varepsilon} \frac{\psi_{D}(j v)}{v} d v
\end{aligned}
$$

where $\psi_{D}(j v)$ is the characteristic function of $D ; \varepsilon$ is a small number to move the path of integration away from the singularity at $v=0$.

Since $D$ is the sum of $L$ non-identically distributed independent variables, the characteristic function of $D$ can be factored into the product of $L$ characteristic functions. Define random variable $d_{k}$ as

$$
d_{k}=X_{k} Y_{k}^{*}+X_{k}^{*} Y_{k}, \quad k=0,1, \cdots, L-1
$$

Since the means of $X_{k}$ and $Y_{k}$ are equal to zero, the characteristic function of $d_{k}$ is given by [4, p.944]

$$
\psi_{d_{k}}(j v)=\frac{v_{k 1} v_{k 2}}{\left(v+j v_{k 1}\right)\left(v-j v_{k 2}\right)}
$$

where the parameters $v_{k 1}$ and $v_{k 2}$ depend on $m_{x_{k} x_{k}}, m_{y_{k} y_{k}}$ and $m_{x_{k} y_{k}}$ similar to (8), which are not identical for different $k$, and we get

$$
\begin{aligned}
& v_{k 1}=\sqrt{w_{k}^{2}+\frac{1}{4\left(m_{x_{k} x_{k}} m_{y_{k} y_{k}}-m_{x_{k} y_{k}}^{2}\right)}}-w_{k}>0 \\
& v_{k 2}=\sqrt{w_{k}^{2}+\frac{1}{4\left(m_{x_{k} x_{k}} m_{y_{k} y_{k}}-m_{x_{k} y_{k}}^{2}\right)}}+w_{k}>0 \\
& w_{k}=\frac{m_{x y}}{2\left(m_{x_{k} x_{k}} m_{y_{k} y_{k}}-m_{x_{k} y_{k}}^{2}\right)}
\end{aligned}
$$

The characteristic function of $D$ is therefore

$$
\psi_{D}(j v)=\prod_{k=0}^{L-1} \psi_{d_{k}}(j v) .
$$

To evaluate the error probability in (11), we derived a partial fraction expansion of $\psi_{D}(j v) / v$ as

$$
\begin{aligned}
& \frac{\psi_{D}(j v)}{v}=\prod_{k=0}^{L-1} \psi_{d_{k}}(j v) \\
= & \frac{1}{v}+(-1)^{L} \sum_{k=0}^{L-1} \frac{v_{k 2}}{v_{k 1}+v_{k 2}} \\
& \times \prod_{l=0, l \neq k}^{L-1} \frac{v_{l 1} v_{l 2}}{\left(v_{k 1}-v_{l 1}\right)\left(v_{k 1}+v_{l 2}\right)} \frac{1}{v+j v_{k 1}} \\
& +(-1)^{L} \sum_{k=0}^{L-1} \frac{v_{k 1}}{v_{k 1}+v_{k 2}} \\
& \times \prod_{l=0, l \neq k}^{L-1} \frac{v_{l 1} v_{l 2}}{\left(v_{k 2}+v_{l 1}\right)\left(v_{k 2}-v_{l 2}\right)} \frac{1}{v-j v_{k 2}}
\end{aligned}
$$

From complex variable theory [6], it follows that

$$
\begin{gathered}
\int_{-\infty+j \varepsilon}^{+\infty+j \varepsilon} \frac{1}{v} d v=0, \int_{-\infty+j \varepsilon}^{+\infty+j \varepsilon} \frac{1}{v+j v_{k 1}} d v=0 \\
\int_{-\infty+j \varepsilon}^{+\infty+j \varepsilon} \frac{1}{v-j v_{k 2}} d v=2 \pi j .
\end{gathered}
$$

The error probability of (11) can be therefore evaluated as

$$
\begin{aligned}
P_{b}= & -\frac{1}{2 \pi j} \int_{-\infty+j \varepsilon}^{+\infty+j \varepsilon} \frac{\psi_{D}(j v)}{v} d v \\
= & (-1)^{L+1} \sum_{k=0}^{L-1} \frac{v_{k 1}}{v_{k 1}+v_{k 2}} \\
& \times \prod_{l=0, l \neq k}^{L-1} \frac{v_{l 1} v_{l 2}}{\left(v_{k 2}+v_{l 1}\right)\left(v_{k 2}-v_{l 2}\right)}
\end{aligned}
$$

\section{Remarks}

- Eq. (18) also gives the BER for MRC with no CE errors in NPDP channels. Fig. 2 shows that the theoretical BER given by (18) is consistent with the simulation results when the PDP have different $\rho=\tau_{0} / \tau_{r m s}$. The circle in the figure denotes the simulation results, which is obtained by semi-analytic simulation method. With the increase of $\rho$, the performance of MRC will degrade due to the severe frequency selective fading. When $\rho$ approach zero, (18) gives the same results as (2). With the increase of the diversity order, the performance degradation due to frequency selectivity becomes more severe.

- Fig. 3 shows the performance loss due to the channel estimation error when $L=4$ under different $\rho$ and $\eta$. The BER can be degraded severely by the channel estimation error. When $\eta=0.5$, the performance loss can be as large as $3 \mathrm{~dB}$.

- The generalization to the $M$-PSK and 2-dimensional RAKE receiver will be given in the journal version of this paper. 


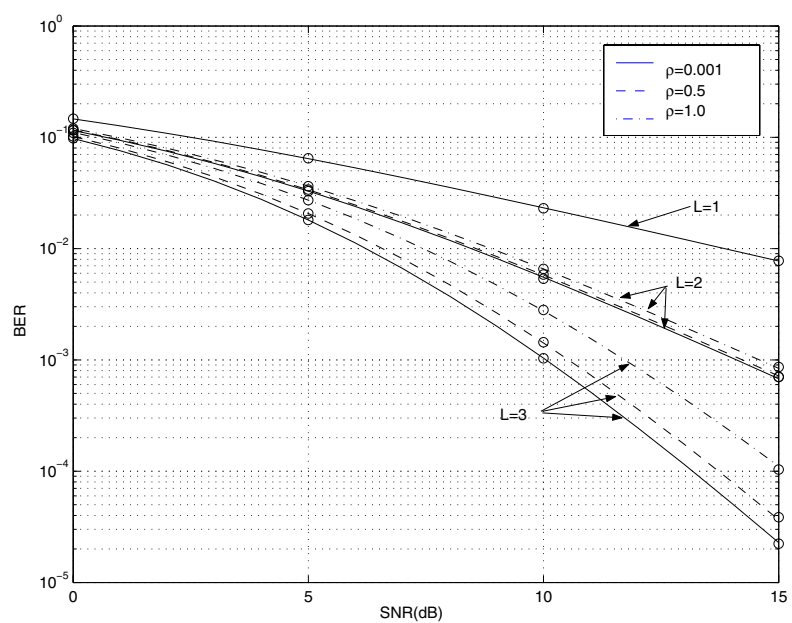

Fig. 2. The BER versus SNR with different $\rho=\tau_{0} / \tau_{r m s}$

\section{OPTIMIZING PILOT SYMBOL LENGTH}

In practical systems, the transmit power within each data frame is fixed and we assume fixed information bit energy to noise ratio $E_{b} / N_{0} . E_{b}$ is the energy per information bit, which is the frame energy divided by the number of information bits. The symbol energy $E_{s}$ relates to $E_{b}$ as $E_{s}=E_{b} N /(N+P)$. For a given fixed $E_{b} / N_{0}$ and channel length, an optimal $P$ exists. This is due to the fact that an increase in $P$ increase the channel estimation accuracy and, thus, decrease the output error probability. On the other hand, given fixed information energy, an increase of the pilot symbol length will decrease the effective symbol SNR and thus, the output error probability will increase.

\section{A. UPDP channels}

Following the derivation of Proakis [4], we can derive the $\mu$ for our LS channel estimator, given by

$$
\mu=\frac{1}{\sqrt{\left(1+R_{s}^{-1}\right)\left(1+\frac{R_{p}^{-1}}{P}\right)}}
$$

where $R_{s}$ is the average output SNR per channel, $R_{p}$ is the average output SNR per pilot bit.

From (2) we can see that the BER monotonically decreases with respect to $\mu$. Therefore, minimizing the BER for BPSK is achieved by maximizing the cross-correlation coefficient $\mu$.

Let $R_{b}=E_{b} E\left(\left|h_{k}\right|^{2}\right) / N_{0}$ is the fixed information symbol energy to noise ratio. Then the average output SNR on each Rake receiver branch is given by $\bar{\gamma}_{c}=R_{b} N /(N+P)$ which is same for all branches. The average output SNR per pilot bit $R_{p}$ is also $R_{b} N /(N+P)$. Then (19) can be written as

$$
\mu=\frac{1}{\sqrt{\left(1+\frac{N+P}{N R_{b}}\right)\left(1+\frac{N+P}{P N R_{b}}\right)}}
$$

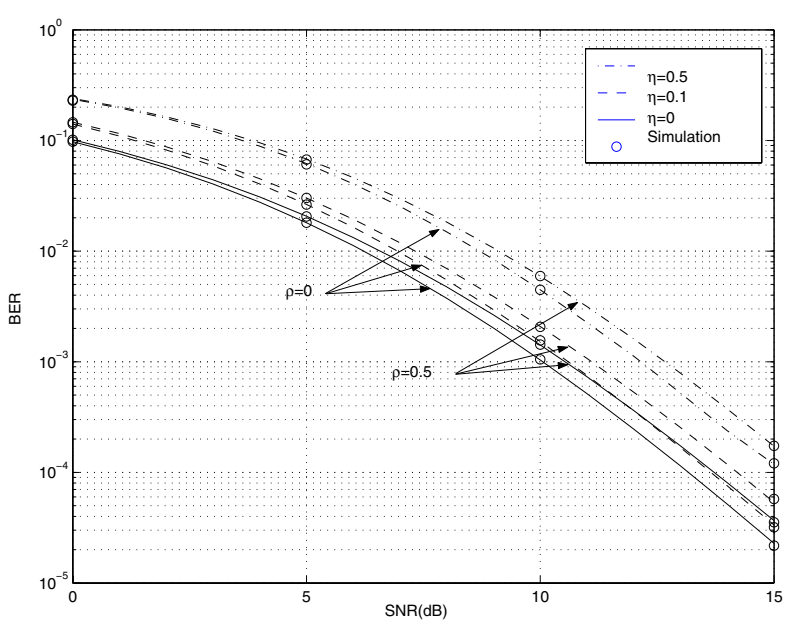

Fig. 3. The BER versus SNR with different $\rho=\tau_{0} / \tau_{r m s}$ and $\eta$

By maximizing (20) with respect to $P$, we find the optimal pilot length to be

$$
P=N \sqrt{\frac{R_{b}}{N R_{b}+1}} \simeq \sqrt{N}
$$

\section{B. NPDP channels}

Like the UPDP channels case, the fixed information symbol average signal-to-noise ratio is defined as

$$
R_{b}=\frac{E_{b}}{N_{0} L} \sum_{k=0}^{L-1} E\left(\left|h_{k}\right|^{2}\right)
$$

The average output SNR of Rake receiver is given by $\bar{\gamma}_{c}=$ $R_{b} N /(N+P)$. Unlike the UPDP case, the average SNR on each Rake receiver branch is different and given by

$$
\bar{\gamma}_{k}=\frac{E_{b} N}{N_{0}(N+P)} E\left(\left|h_{k}\right|^{2}\right)
$$

Unfortunately, as (18) is complicated, a closed-form expression for the optimal pilot symbol length can not be derived. Instead, we find the optimal pilot length from simulation.

\section{SIMULATION RESULTS}

The optimal pilot length versus the number of data symbols $N$ for a 3 paths UPDP channel with different information symbol SNR is given in Fig. 4. Fig. 5 shows the BER versus the information symbol SNR when using the optimal number of pilot symbols for each value of SNR and when using $P=6$ and 50, with data length $N=200$ and 3 paths EPDP channel.

Next, we investigate the optimal pilot length for NPDP frequency selective channels. Our channel model has an exponential power delay profile as (3). Fig. 6 shows the optimal pilot length versus the number of data symbols $N$ for a 3 paths EPDP channel with different information symbol SNR. The optimal pilot length is still $\sqrt{N}$ in high SNR region while it is not true in low SNR. Fig. 7 shows the BER versus the information symbol SNR when using the optimal number 


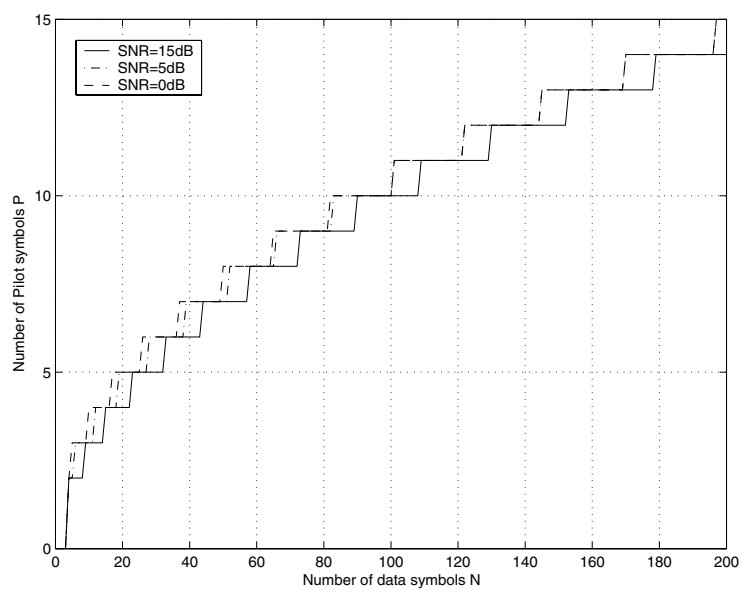

Fig. 4. The optimal pilot length versus bit error probability, when $L=3$, UPDP

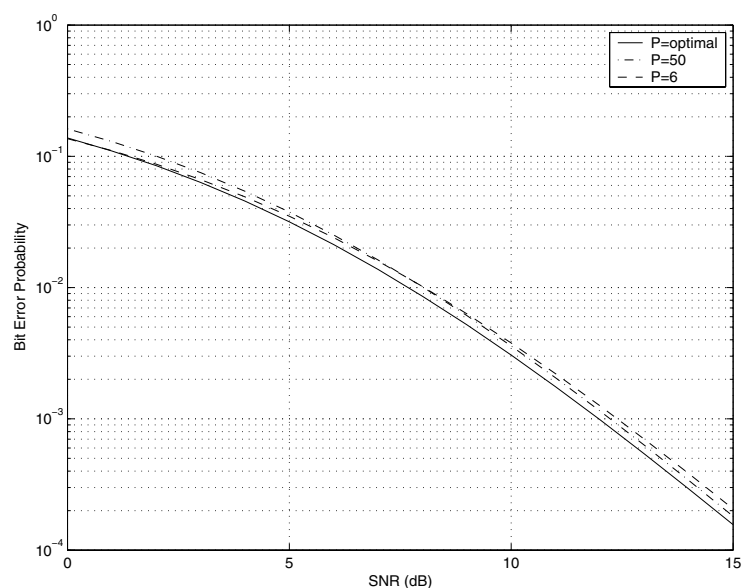

Fig. 5. The BER versus the information symbol SNR, when $L=3$, UPDP

of pilot symbols in $15 \mathrm{~dB}$ for each value of SNR and when using $P=6$ and 50, with data length $N=200,3$ paths EPDP channel. This figure clearly shows that the optimal pilot length in high SNR is not optimal in low SNR.

\section{CONCLUSION}

We have investigated the optimization of pilot sequences for general DS-CDMA systems in slow fading frequency selective Rayleigh channel. The effects of imperfect CE have been analyzed for a MRC RAKE receiver in both UPDP and NPDP channels. We derive the closed-form of the BER for RAKE receiver in NPDP channels through a different approach from Proakis [4]. In a practical system, the energy within each data frame is constant. Thus, we optimize the length of the pilot symbols by minimizing the bit error probability of the MRC receiver. We find the optimal length of the pilot symbols is the square root of the information data length in UPDP channels and this relation is only true for NPDP channels in high SNR. The results of the paper is useful to design a pilot-symbolassisted modulation system.

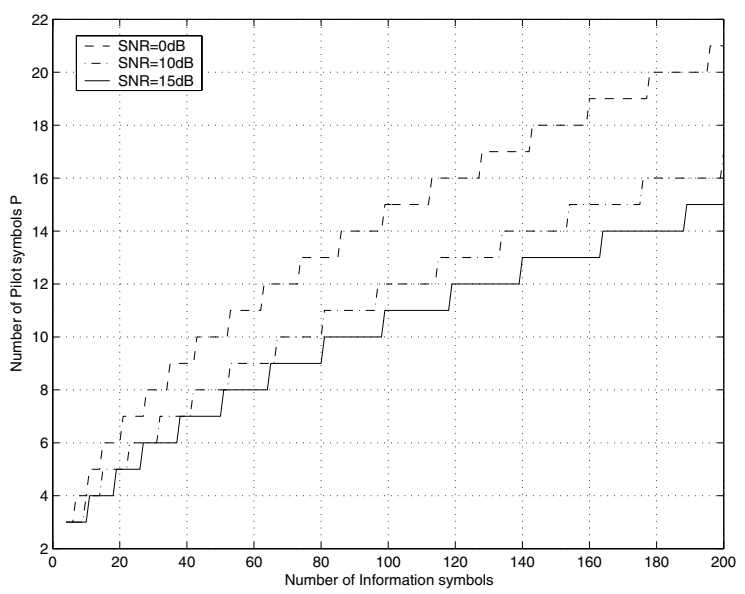

Fig. 6. The optimal pilot length versus bit error probability, when $L=3$, EPDP

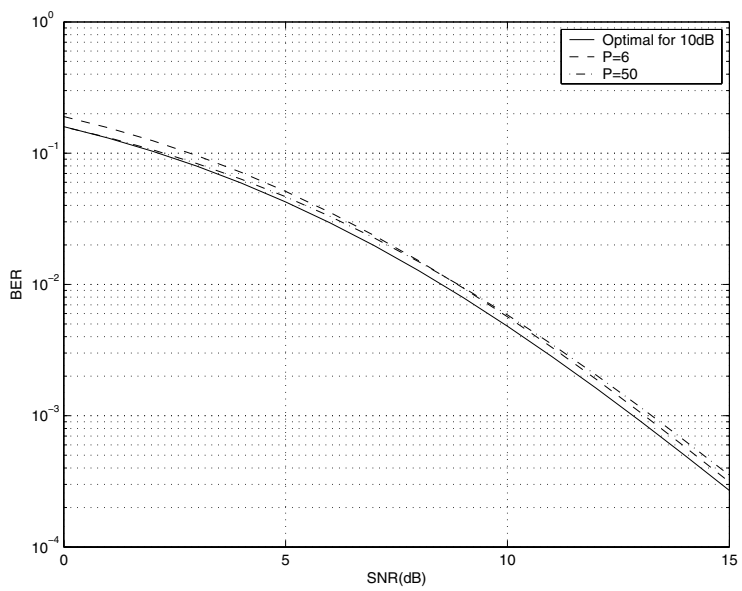

Fig. 7. The BER versus the information symbol SNR, when $L=3$, EPDP

\section{REFERENCES}

[1] P. Chaudhury, W. Mohr, and S. Onoe, "The 3GPP proposal for IMT2000," IEEE Communications Magazine, vol. 37, no. 12, pp. 72-81, Dec. 1999.

[2] M. Gans, "The effect of Gaussian error in maximal ratio combiners," IEEE Transactions on Communications, vol. 19, no. 4, pp. 492-500, Aug. 1971.

[3] B. Tomiuk, N. Beaulieu, and A. Abu-Dayya, "General forms for maximal ratio diversity with weighting errors," IEEE Transactions on Communications, vol. 47, no. 4, pp. 488-492, Apr. 1999.

[4] J. G. Proakis, Digital Communications, 4th ed. New York: McGraw-Hill, 2001.

[5] D. Singh, A. Kumari, R. Mallik, and S. Jamuar, "Analysis of rake reception with MRC and imperfect weight estimation for binary coherent orthogonal signaling," IEEE Communications Letters, vol. 6, no. 6, pp. 245-247, Jun. 2002.

[6] L. Serge, Complex analysis, 3rd ed. New York : Springer-Verlag, 1993. 